\title{
DEFINIÇÕES E DIAGNÓSTICO DA EJACULAÇÃO RÁPIDA
}

Bruno Silva Peixoto de Carvalho ${ }^{1}$; Gisele Vajgel Fernandes ${ }^{2}$; Adriano Almeida Calado ${ }^{3}$

\section{DEFINITIONS AND DIAGNOSIS OF RAPIDE EJACULATION}

Resumo: Com este artigo, os autores procuram estudar as definições e diagnósticos de uma das disfunções mais freqüentes na sexualidade humana: a ejaculação rápida (E.R.). Através de extensa revisão bibliográfica, diversas teorias e métodos foram abordados e, de uma maneira simplificada e de fácil compreensão, apresentados aos leitores. Após uma introdução necessária para melhor familiarização com o problema, foram discutidas as diversas definições existentes para esta condição tão importante para a satisfação sexual dos casais. Mostrou-se também algumas vertentes de pensamento para a etiologia da ejaculação rápida, dos estudos mais tradicionais até os mais recentes artigos publicados.E para finalizar foi apresentado o estado da arte no diagnóstico, detalhando a avaliação das ferramentas mais utilizadas para a formatação de ensaios clínicos, avaliação diagnóstica e seguimento terapêutico.

Palavras-chave: Ejaculação; definição; diagnóstico.

Abstract: This article look forward to study the definitions and diagnosis of premature ejaculation (P.E.), one of the most frequent sexual disorder nowadays. Through an extense literature review, the authors studied a serie of theories and methods, and in a simplified way, presented them to the journal readers. After an necessary introduction, some differents definitons of P.E. were showed and discussed to help the understanding of such an

1 Mestrando em ciências médicas pela Universidade de Pernambuco.

e-mail: brunocarvalho@prontomail.com

2 Mestranda em ciências médicas pela Universidade de Pernambuco.

e-mail: giselevajgel@hotmail.com

3 Doutor em urologia pela Unifesp.

e-mail: caladourologia@yahoo.com.br 
important condition to couple's sexual satisfaction. Also some etiologies trains of thought were shown, from the very early studies to the most recent articles published in the indexed literature. To finish, the state of art in the diagnostical tools was presented, in a effort to maximize clinical trials findings and clarify the use of self-applicated outcome questionnaires.

Keywords: Ejaculation; definition; diagnosis.

\section{Introdução}

A ejaculação rápida (E.R.) é uma disfunção sexual muito importante, pois pode afetar a satisfação sexual tanto do homem como de sua parceira(o). Contudo dispomos de poucos estudos, de nível elevado de evidência, que estudaram o real impacto da E.R. no homem, sua parceira(o) e no relacionamento global do casal (ROWLAND, COOPER, SCHNEIDER, 2001; BYERS, GRENIER, 2003). A disfunção ejaculatória é provavelmente a desordem sexual mais freqüente na população masculina. E com certeza a E.R. é a mais prevalente das disfunções ejaculatórias. Porém, ainda assim, não há um consenso em relação a uma definição universalmente aceita. (LAUMANN, PAIK, ROSEN, 1999). Temos uma variedade muito grande de estimativas de prevalência, como também uma série de proposições terapêuticas sem a necessária normatização (ASSALIAN, 2005).

Aqui em nosso meio, nota-se uma hesitação por parte de certa porcentagem dos pacientes em procurar ajuda profissional, ou até mesmo falar sobre o assunto com o seu médico generalista. Talvez por falta de um bom relacionamento médico-paciente ou apenas a inexistência de um tratamento popularmente conhecido e eficaz, um Viagra ${ }^{\circledR}$ para ejaculação precoce por exemplo.

Em alguns relacionamentos o problema é contornado através de diversos mecanismos, acarretando um menor grau de incômodo. Entretanto a E.R. pode causar sérios distúrbios ao relacionamento, surgindo até acusaçōes de egoísmo e comportamentos anormais, como o de evitar as atividades sexuais. E isso tudo, obviamente deixa a condição ainda mais grave.

\section{Fisiologia Ejaculatória}

A ejaculação é um fenômeno que envolve tanto o sistema nervoso central (S.N.C.) quanto o sistema nervoso periférico (S.N.P.). Existem várias áreas 
do S.N.C. envolvidas na ejaculação - cérebro,tronco cerebral e medula espinhal. Estudos recentes utilizando a tecnologia de emissão de pósitrons (Petscan) evidenciou grande estimulação da área tegumentar ventral durante o processo ejaculatório. (HOLSTEGE, GEORGIADIS, PAANS, 2003)

Vale ressaltar que a ejaculação é dividida em duas fases distintas: Emissão e expulsão. Na emissão o sêmen é depositado na uretra posterior e o esfíncter interno da bexiga se fecha. $\mathrm{Na}$ expulsão, que segue imediatamente à emissão, o sêmen é impulsionado pela uretra por contrações da musculatura do assoalho pélvico. No processo ejaculatório estão envolvidos tanto mediadores serotominérgicos quanto dopaminérgicos. Sabe-se que a interferência nos mecanismos de regulação serotominérgicos (dessensibilização dos receptores 5-HT), só acontece após uma ou duas semanas de uso diário de drogas, como a maioria dos inibidores seletivos da recaptação da serotonina. (WALDINGER, SCHWEITZER, OLIVIER, 2005) Este fenômeno tem que ser considerado na conduta farmacológica nestes casos.

\section{Epidemiologia e definições}

Considera-se a ejaculação rápida a mais comum das disfunções sexuais masculinas, afetando de 5 a 40\% dos homens sexualmente ativos (ST. LAURENCE, MADAKASIRA, 1992). Existe uma idéia que isto aconteça mais comumente em jovens, mas existe E.R. em todas as faixas etárias. A distribuição também pode variar geograficamente (JANNINI, LENZI, 2005). Uma questão a ser resolvida é a dificuldade de avaliar as queixas subjetivas de E.R.. Fatores culturais e de grau de emancipação feminina dificultam ainda mais esta análise. Atualmente consideram-se dois tipos de E.R.: a primária (aquela que existe desde os primeiros intercursos sexuais) e a adquirida (aquela que surge durante a vida sexual previamente normal). Através de busca na literatura encontra-se diversos termos e definições para E.R., esta falta de consenso prejudica toda a pesquisa e conduta nesta condição. Masters \& Johnson a definiu em 1970 como a inabilidade de atrasar a ejaculação o suficiente para que a mulher atinja o orgasmo em pelo menos $50 \%$ das tentativas. A Associação Psiquiátrica Americana (A.P.A.) a classificou como ejaculação após mínimo estímulo, antes durante ou logo após a penetração, sem que o homem a deseje, de forma persistente ou recorrente. Também faz-se mister que isto acarrete angústia ou dificuldade de relacionamento.

A Organização Mundial da Saúde (O.M.S.) a define como a impossibilidade de adiar a ejaculação o suficiente para não aproveitar a relação sexual. Um 
estudo multicêntrico, realizado em 2005, propôs que homens com um tempo de latência ejaculatória intra-vaginal (I.E.L.T.) menor que 0,9 minutos teriam E.R. e que aqueles com I.E.L.T. entre 0,9 e 1,3 minutos seriam classificados como prováveis portadores de E.R. (WALDINGER, QUINN, DILEEN, 2005). Esta seria uma das primeiras tentativas de definir a E.R. com instrumentos mais fidedignos, porém mais estudos precisam ser desenvolvidos.

\section{Etiologia}

Existem diversas explicaçôes plausíveis para a gênese da E.R.. Há décadas que acreditamos que este fenômeno é fruto de fatores psicológicos. Alguns modelos foram propostos. Em 1917, Abraham sugeriu que a E.R. acontece graças a sentimentos negativos contra a parceira (ABRAHAM, VEBER, 1917) Já me 1943, Schapiro aventou que a E.R. tivesse origem em problemas psicológicos que teriam influência na atividade genital (SCHAPIRO, 1943) Contudo não há estudos comprovando as hipóteses destes dois autores. Mais recentemente na década de 70, estudo clássico de Masters \& Johnson referia que a E.R. seria conseqüência de problemas ocorridos no primeiro intercurso sexual (MASTER, JOHNSON, 1970) Kaplan, em 1974, propôs que o fator etiológico central da E.R. seria a falta de consciência do nível de excitação e dos sinais pré-orgásmicos (KAPLAN, 1974). Porém não há provas fortes destas duas hipóteses.

Em 1988, Assalian sugeriu uma hipersensibilidade no sistema nervoso simpático (ASSALIAN, 1988), e finalmente em 1998, Waldinger postulou que o I.E.L.T. teria uma variação biológica decorrente de uma desregulação da ativação de alguns sub-tipos dos receptores serotominérgicos (WALDINGER, BERENDSEN, BLOK, OLIVIER, HOLSTEGE, 1998). Já existem estudos, em animais e humanos, que demonstram claramente uma distribuição matemática deste I.E.L.T., e o papel central do sistema serotominérgico no processo ejaculatório. (WALDINGER, QUINN, DILLEEN, 2005; PATTIJ, DE JONG, UITTERDIJK, A., 2005). Para a E.R. adquirida pouca atenção foi dada pelos pesquisadores. Temos três causas freqüentemente citadas (WALDINGER, ZWINDERMAN, OLIVIER, SCHWEITZER, 2005):

- Problemas urológicos como a disfunção erétil e prostatites;

- Problemas psicológicos como o estresse de um novo relacionamento;

- Problemas endócrinos como a disfunção de tiróide, notadamente o hipertiroidismo. 


\section{Diagnóstico}

Se a gênese da E.R. ainda não está totalmente evidenciada, ao menos o diagnóstico é bem menos complexo. Baseia-se no relato do paciente, história clínica e exame físico. Devemos coletar uma história detalhada, tanto clínica como sexual, e realizar um exame físico padrão, visando detectar alguma possível causa orgânica eventual. Algumas armadilhas são comuns nestes casos. Homens sem E.R. tendem a superestimar seu I.E.L.T. comparando com homens com E.R.. E nestes homens com E.R. o tempo medido por cronômetros, em geral, confere com o tempo por eles estimado. Isto sugere que o relato da estimativa do I.E.L.T., pelo paciente, é suficiente para a confirmação diagnóstica na prática cotidiana. Também é importante, diferenciar o tipo de E.R. enfrentado, se primário ou adquirido. Nesta última, os sintomas tendem a ser situacionais. Um problema conceitual se coloca. Precisamos, para estudos de avaliação de sucesso terapêutico, ter ferramentas matemáticas e questionários confiáveis para uma análise mais precisa. (PRYOR, BRODERICK, HO, JAMIESON, GAGNON, 2005; SYMONDS, ALTHOF, ROSEN, ROBLIN, LAYTON, 2002; YUAN, XIN, JIANG, 2004; RUST, GOLOMBOK, 1986)

Sabemos que o relato do I.E.L.T. confere substancialmente com o tempo cronometrado. Mas não temos a mesma convicção nesta relação quando analisamos o aumento deste tempo pós-tratamento. Apresentamos a seguir algumas das medidas que podem ser utilizadas em ensaios clínicos:

- I.E.L.T., definido como o tempo entre a penetração e a ejaculação. É medido pela parceira com cronômetros eé utilizado na maioria dos estudos recentes. Não há uma definição do ponto de corte para diagnosticar a E.R. Os interessados podem acessar o Journal of sexual Medicine e ler o artigo de Waldinger et al. com algumas sugestões de valores (WALDINGER et al., 2005)

- Controle Voluntário (C.V.), alguns autores proporam o C.V. como um bom medidor para definição da E.R (KAPLAN, KOHL, POMEROY, OFFIT, HOGAN, 1974; MCCARTHY, 1988; VANDEREYCKEN, 1986). Mas achamos este muito subjetivo e de difícil quantificação.

Algumas tentativas de medir esta capacidade de C.V. foram realizadas (GRENIER, BYERS, 1997; PATRICK, ALTHOF, PRYOR, 2005; WALDINGER, BERENDSEN, BLOK, OLIVER, HOLSTAGE, 1998), porém não se encontrou diferenças entre os homens com ou sem E.R. 
- Incômodo, o incômodo causado pela E.R. é uma variável bastante importante a se estudar. A sua extensão pode definir a gravidade da E.R. (PATRICK et al., 2005; PRYOR et al., 2006; LUE, BASSON, ROSEN, 2004).

- Satisfação sexual, pacientes com E.R., sistematicamente relatam ter menor satisfação sexual se comparados aos homens que não tem E.R. (PATRICK et al., 2005).

\section{Mensagem Final}

A ejaculação rápida é um desafio para todos que militam na área da sexualidade humana. Cada vez mais devemos envidar esforços para esclarecer as dúvidas existente agora e as que, com certeza, surgirão no futuro.

\section{Referências bibliográficas}

ABRAHAM, K.;. UEBER Ejaculatio Praecox. Zeitschr Aerztl Psychoanal; 4: 171, 1917.

ASSALIAN, P. Guidelines for the pharmacotherapy of premature ejaculation. World J Urol; 12: 12, 2005.

BYERS, E.S.; GRENIER, G. Premature or rapid ejaculation: heterosexual couple's perceptions of men's ejaculatory behavior. Arch Sex Behav; 32 (3): 261-270, 2003.

GRENIER, G.; BYERS, E.S.; The relationship among ejaculatory control, ejaculatory latency and attempts to prolong heterosexual intercourse. Arch Sex Behav; 26 (1): 27-47, 1997.

HOLSTEGE, G., GEORGIADIS, J.R.; PAANS, A.M. et al Brain activation during human male ejaculation. J Neurosci; 23: 9185-8193, 2003.

JANNINI, E.A.; LENZI, A. Ejaculatory disorders: epidemiology and current approaches to definitions, classification and subtyping. World J Urol; 18:18., 2005.

KAPLAN, H.S. The new sex therapy. New York: Brunner/Mazel, 1974. KAPLAN, H.S.; KOHL, R.N.; POMEROY, W.B.; OFFIT, A.K.; HOGAN, B. Group treatment of premature ejacutation. Arch Sex Behav; 3 (5): 443-452, 1974.

LAUMANN, E.O.; PAIK, A.; ROSEN, R.C. Sexual dysfunction in the United States: prevalence and predictors. Jama, 281 (6): 537-544, 1999. LUE, T.F.; BASSON, R.; ROSEN, R.C. Sexual medicine dysfunctions in men and women. 2004 
MASTERS, W.H.; JOHNSON, V.E. Human Sexual Inadequacy. Boston: Little brown, 1970, pp 92-115.

MCCARTHY, B. Cognitive behavioural strategies and techniques in the treatment of early ejaculation. In: LEIBLUM, S.R.; ROSEN, R.C. eds. Principles and practices of sex therapy: Update for the 1990s. Guildford Press: New York, 1988, pp.141-167.

PATRICK, D.L.; ALTHOF, S.E.; PRYOR, J.L. Premature ejaculation: An observational study of men and their partners. J Sex Med; 2(3): 358367, 2005.

PATTIJ, T.; DE JONG, T.R. et al.? Individual differences in male rat ejaculatory behavior : searching for models to study ejaculatory disorders. Eur J Neurosci; e-pub., 2005.

PORST, H.; BUVAT, J. Standard Practices in Sexual Medicine, Blackwell Publishing, Massachusetts, USA: 2006.

PRYOR, J.L.; BRODERICK, G.A.; HO, K.F.; JAMIESON, C.; GAGNON, D. Comparison of estimated vesus measured intravaginal ejaculatory latency time (IELT) in men with and without premature ejaculation (PE) In: PORST, H.; BUVAT, J. Standard Practices in Sexual Medicine, Blackwell Publishing, Massachusetts, USA: 2006.

ROWLAND, D.L.; COOPER, S.E.; SCHENEIDER, M. Defining premature ejaculation for experimental and clinical investigations. Arch Sex Behav; 30 (3): 235-253, 2001.

RUST, J.; GOLOMBOK, S. The GRISS: a psychometric instrument for the assessment of sexual dysfunction. Arch Sex Behav; 15(2): 157165, 1986.

SCHAPIRO, B. Premature ejaculation, a review of 1130 cases. J Urol; 50: 374-379, 1943.

ST. LAWRENCE, J.S.; MADAKASIRA, S. Evaluation and treatment of premature ejaculation: a critical review. Int J psychiatr Med; 22: 77, 1992.

SYMONDS, T.; ALTHOF, S.E.; ROSEN, R.C.; ROBLIN, D.; LAYTON, M. Questionnaire assessment of ejaculatory control: development and validation of a new instrument. Int J Imp Res; 14 (suppl 4): S33: abstract PS - 7-1, 2002.

VANDEREYCKEN, W. Towards a better delineation of ejaculatory disorders. Acta Psychiatr Belg; 86 (1): 57-63, 1986.

WALDINGER, M.D.; BERENDSEN, H.H.; BLOK, B.F.; OLIVIER, B.; HOLSTAGE, G. Premature ejaculation and serotoninergic antidepressantes-induced delayed ejaculation: the involvement of the serotonergic system. Behav Brain Res; 92 (2): 111-118, 1998. 
WALDINGER, M.D.; HENGELVER, ZWINDERMAN, A.H.; OLIVIER, B. An empirical operationalization of DSM-IV diagnostic criteria for premature ejaculation. Int J Psychiatry Clin Pract; 2: 287-293, 1998. WALDINGER, M.D.; QUINN, P.; DILLEEN, M.; et al.. A multinational population survey of intravaginal ejaculation latency time. J Sex Med; 2: 492-497, 2005.

WALDINGER, M.D.; SCHWEITZER, D.H.; OLIVIER, B. On demand SSRI treatment of premature ejaculation: pharmacodynamic limitations for relevant ejaculation delay and consequent solutions. $\mathbf{J}$ Sex Med; 2 : 121-131, 2005.

WALDINGER, M.D.; ZWINDERMAN, A.H.; OLIVIER, B.; SCHWEITZER, D.H. Thiroid-stimulating hormone assessments in a Dutch cohort of 620 men with lifelong premature ejaculation without erectile dysfunction. J Sex Med; 2 (6): 865-870, 2005.

WALDINGER, M.D.; ZWINDERMAN, A.H.; OLIVIER, B.; SCHWEITZER, D.H. Proposal for a definition of lifelong premature ejaculation based on epidemiological stopwatch data. J Sex Med; 2 (4): 498-507, 2005.

WALDINGER, M.D.; ZWINDERMAN, A.H.; OLIVIER, B.; SCHWEITZER, D.H. Relevance of methodological design for the interpretation of efficacy of drug treatment of premature ejaculation: a systematic review and meta-analysis. Int J Impot Res; 16 (4): 369381, 2004.

YUAN, Y.M.; XIN, Z.C.; JIANG, H. et al. Sexual function of premature ejaculation patients assayed with Chisese Index of Premature Ejaculation. Asian J Androl; 6 (2): 121-126, 2004. 